\title{
Relationship between Ocular Pain and Optic Tract Involvement in Optic Neuritis Analyzed by Magnetic Resonance Imaging
}

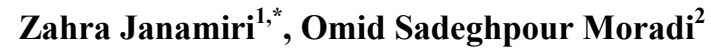 \\ ${ }^{1}$ Department of Radiology Technology, Shohada Tajrish Hospital, School of Allied Medicl Sciences, \\ Shahid Beheshti University of Medical Sciences, Tehran, Iran \\ ${ }^{2}$ Shahid Beheshti University of Medical Sciences, Tehran, Iran \\ *Corresponding author: Zahra.janamiri@gmail.com
}

\begin{abstract}
Introduction: Optic Neuritis is defined as inflammation of the optic nerve, which is mostly idiopathic, while demyelinating lesions secondary to auto-immune conditions are among other causes. These lesions are demonstrated on Magnetic Resonance Imaging (MRI) as hyper intensities. In this study, authors plan to investigate the relationship between MRI pattern of patients suffering from optic neuritis, and their pain and visual loss patterns. Materials and Methods: From June 2017 to December 2017, we have enrolled 127 patients suffering from optic neuritis. These patients were referred to neurology and ophthalmology clinic of a private hospital in Tehran. Demographic data of all patients were collected and they underwent MRI for evaluation of optic nerve abnormalities and possible lesions and enhancements. Also, detailed pain score regarding their ocular pain were recorded by an expert blind neurologist, categorizing them into pain with movement, pain during rest and no pain. Also, visual field assessments were performed by an expert blinded ophthalmologist. Statistical analysis performed using SPSS version 17.00 and Student T-test and Mann-whitney test and Chi-square test. Results: There were 110 females and 17 males, with mean age of 35.2 years for females and 39.8 for males. Statistical analysis revealed no significant difference between demographic data of patients. ( $\mathrm{P}$ value $=0.12)$ Of these patients, 116 cases have abnormalities in their neuro-imaging, which 60 cases have pain in eye movement (group A), 37 cases experienced pain during rest (group B) and 19 cases have no pain (group C). Also, 78 patients have optic nerve enhancement in the orbital segment (39 cases of group A, 28 cases of group B, 11 cases of group C), 16 patients have canalicular segment enhancement ( 4 cases of group A, 9 cases of group B, 3 cases of group C) and 22 patients have intracranial segment enhancement (13 cases of group A, 6 cases of group B, 3 cases of group C). Also we have found that enhancements longer than $8 \mathrm{~mm}$ are consistent with more severe ocular pain, whether during movement or rest. $(\mathrm{P}$ value $<0.05)$ Visual field assessment revealed no significant correlation between type and length of optic nerve enhancement on MRI and visual field loss. (P value $=0.09$ ). Conclusion: Authors have found that most of the patients suffering from optic neuritis may demonstrate their symptoms as ocular pain during movement, which most of them have orbital segment involvement of optic nerve. Also, we have found that canalicular optic nerve enhancement were mostly associated with ocular pain at rest, opposed to other types of enhancement (orbital and intracranial). Also, we have found a specific threshold defined as $8 \mathrm{~mm}$ of optic nerve enhancement and severity of pain, which is less than other studies in literature.
\end{abstract}

Keywords: optic neuritis, magnetic resonance imaging, enhancement, optic nerve length, visual field

Cite This Article: Zahra Janamiri, and Omid Sadeghpour Moradi, "Relationship between Ocular Pain and Optic Tract Involvement in Optic Neuritis Analyzed by Magnetic Resonance Imaging." American Journal of Medical Case Reports, vol. 6, no. 2 (2018): 33-35. doi: 10.12691/ajmcr-6-2-6.

\section{Introduction}

Optic neuritis is defined as the inflammation of optic nerve, which is mostly manifested as sub-acute unilateral painful visual loss mostly in a young healthy female. [1] It is mostly due to demyelinating lesions such as multiple sclerosis and neuromyelitis optica, and less commonly due to autoimmune disease such as sarcoidosis and systemic lupus erythematosus, infectious causes such as syphilis and tuberculosis, inflammatory and post vaccination immunological responses such as sinusitis and vaccinations against measles and rubella. [2] Female to male ratio is 3 to 1 and annual incidence of optic neuritis is reported as 6 per 100000. [3] The exact pthophysiological mechanism is not well understood, but previous studies and documents proposed a type 4 hyper-sensitivity reaction induced by released cytokines and other inflammatory mediators from activated peripheral T-cells capable of targeting myelin sheets of optic nerve. [4] Patients may experience ocular pain, visual field loss, 
Uhthoff's phenomenon defined as exercise or heat-induced deterioration of visual symptoms, Pulfrich phenomenon defined as misperception of the direction of movement of an object, optic disc pallor and photopsia. [5] In this study, authors plan to investigate the relationship between MRI pattern of patients suffering from optic neuritis, and their pain and visual loss patterns.

\section{Materials and Methods}

From June 2017 to December 2017, we have enrolled 127 patients suffering from optic neuritis were referred to neurology and ophthalmology clinic of a private hospital in Tehran. Authors recorded demographic data such as age, sex, ethnicity, initiation of pain, initiation of visual loss, underlying auto-immune or auto-inflammatory or collagen-vascular diseases and malignancies, drug history and previous surgical and radiation treatment received for the head and neck and intracranial problems. Patients with previous history of intracranial malignancies, history of cranial or scalp radiation therapy, recent ocular trauma, underlying ocular diseases, underlying collagen-vascular diseases and patients with insufficient demographic data and who did not signed the written informed consent were excluded from the study. Also, we have performed MRI for assessment of possible optic nerve lesions and their enhancement patterns. Detailed pain score regarding their ocular pain were recorded by an expert blind neurologist, categorizing them into pain with movement, pain during rest and no pain. Patients underwent visual field assessment for documentation of their visual loss and examining the possible occult visual field defect not perceptible to the patient by an expert blind ophthalmologist. Statistical analysis performed using SPSS version 17.00 and Student T-test and Mann-whitney test and Chi-square test. Patients entered to the study after collection of written informed consent authorized by ethics committee of our private hospital based on Helsinki Ethics Declaration.

\section{Results}

We have enrolled 127 patients, including 110 females and 17 males, with mean age of 35.2 years for females and 39.8 for males of Iranian population referring to neurology and ophthalmology clinic of our hospital. No significant difference was observed between demographic data range of our patients. ( $\mathrm{P}$ value $=0.12$ ) After performing neuro-imaging examination by MRI, 11 patients were excluded from the study (secondary exclusion) due to absence of lesion enhancement of optic nerve in their MRI. Of 116 remaining patients with abnormalities such as enhancement and lesions in their optic nerve (excluding tumors), 60 cases $(51.7 \%)$ have pain in eye movement (group A), 37 (31.8\%) cases experienced pain during rest (group B) and 19 cases (16.3\%) have no pain (group C). Also, 78 patients $(67.2 \%)$ have optic nerve enhancement in the orbital segment (39 cases of group A $(50 \%), 28$ cases of group B $(35.8 \%)$, 11 cases of group C (14.1\%)), 16 patients $(13.7 \%)$ have canalicular segment enhancement
(4 cases of group A (25\%), 9 cases of group B (56.2\%), 3 cases of group $\mathrm{C}(18.8 \%))$ and 22 patients $(18.9 \%)$ have intracranial segment enhancement (13 cases of group A $(59 \%), 6$ cases of group B $(27.2 \%), 3$ cases of group C $(13.6 \%))$. Also we have found that enhancements longer than $8 \mathrm{~mm}$ are consistent with more severe ocular pain, whether during movement or rest. ( $\mathrm{P}$ value $<0.05)$ Visual field assessment revealed no significant correlation between type and length of optic nerve enhancement on MRI and visual field loss. (P value=0.09).

\section{Discussion}

Multiple sclerosis and neuromyelitis optica are among the two most common causes of optic neuritis among population, especially among female population with 3 to 1 prevalence to male. [6] However, there are other conditions mimicking optic neuropathy, including Anterior Ischemic Optic Neuropathy (AION) which occurs in patients aged more than 50 years, have same prevalence among males and females and is characterized by unilateral sudden painless visual loss. [7] Also, another condition mimicking optic neuritis and should be in our differential diagnosis list, is Leber's Hereditary Optic Neuropathy (LHON) is a mitochondrial genetic disorder which manifest itself as sub-acute and painless visual loss with central scotoma and it is predominately affects $80 \%$ to $90 \%$ of young males. [8] There are four types of optic neuritis based on anatomical location of the lesion: 1) retrobulbar neuritis with normal disc, 2) papillitis with swollen disc, 3) perineuritis with or without optic disc involvement and 4) neuroretinitis with optic disc edema. $[9,10]$ Diagnosis of optic neuritis is based on thorough neurological examination, focused on cranial nerve II, III, IV and VI for light perception, pupillary reflex and ocular movements, as well as cranial nerve $\mathrm{V}$ for pain perception in trigeminal nerve territory especially in V1 (ophthalmic branch). Also, ophthalmic examinations including slit lamp examination and pupillary reactions (RAPD) is included in ophthalmic evaluation of both eyes. Although diagnosis is established in most of the cases, neuro-imaging modalities such as MRI with or without Gadolinium contrast can improve diagnosis accuracy and is useful in follow up assessment and evaluation of extent of involvement. [11,12] MRI may demonstrate hyper intense lesions in optic nerve, and may be appreciated by Gadolinium contrast. Visual field assessment is also useful in assessment of visual field deficits and occult and hidden blind spots in visual field which patients may not be aware of. Steroid and immune-modulator therapy is recommended for treatment of optic neuritis. [13] In this study, we have found a specific limit for severity of pain, defined as $8 \mathrm{~mm}$ of optic nerve enhancement, while Fazzone et al have reported this limit as $10 \mathrm{~mm}$. [14] Also, we have demonstrated relationship between ocular pain patterns and segment of optic nerve involvement, which may be considered in optimal treatment of these patients based on anatomical lesion, and also will increase the accuracy of diagnosis of possible anatomical location of lesion on optic tract in case of absence of neuro-imaging modalities, in more remote areas. 


\section{Conclusion}

Authors have found that most of the patients suffering from optic neuritis may demonstrate their symptoms as ocular pain during movement, which most of them have orbital segment involvement of optic nerve. Also, we have found that canalicular optic nerve enhancement were mostly associated with ocular pain at rest, opposed to other types of enhancement (orbital and intracranial). Also, we have found a specific threshold defined as $8 \mathrm{~mm}$ of optic nerve enhancement and severity of pain, which is less than other studies in literature.

\section{References}

[1] Beck, R.W., Smith, C.H., Gal, R.L., Xing, D., Bhatti, M.T. Brodsky, M.C., Buckley, E.G., Chrousos, G.A., Corbett, J., Eggenberger, E., Goodwin, J.A., "Neurologic impairment 10 years after optic neuritis," Archives of neurology, 61(9). 1386-9. 2004

[2] Voss, E., Raab, P., Trebst, C., Stangel, M., "Clinical approach to optic neuritis: pitfalls, red flags and differential diagnosis," Therapeutic advances in neurological disorders, 4(2). 123-34. 2011.

[3] Flanagan, P., Zele, A.J., "Chromatic and luminance losses with multiple sclerosis and optic neuritis measured using dynamic random luminance contrast noise," Ophthalmic and Physiological Optics, 24(3). 225-33. 2004.

[4] Wingerchuk, D.M., Weinshenker, B.G., "Neuromyelitis optica," Current treatment options in neurology, 10(1). 55-66. 2008.

[5] Frederiksen, J.L., Larsson, H.B., Henriksen, O., Olesen, J., "Magnetic resonance imaging of the brain in patients with acute monosymptomatic optic neuritis," Acta neurologica scandinavica, 80(6). 512-7. 1989.

[6] Rizzo, J.F., Lessell, S., "Optic neuritis and ischemic optic neuropathy: overlapping clinical profiles," Archives of Ophthalmology, 109(12). 1668-72. 1991.

[7] Hayreh, S.S., "Management of ischemic optic neuropathies," Indian journal of ophthalmology, 59(2). 123. 2011

[8] Newman, N.J., "Treatment of Leber hereditary optic neuropathy," Brain, 134(9). 2447. 2011

[9] Trobe, J.D., Sieving, P.C., Guire, K.E., Fendrick, A.M., "The impact of the optic neuritis treatment trial on the practices of ophthalmologists and neurologists," Ophthalmology, 106(11). 2047-53. 1999.

[10] Seddighi, A., Akbari, M.E., Seddighi, A.S., Pirayesh, E., Soleymani, M.M., Baqdashti, H.R., Nikouei, A., Zali, A., Tabatabaei, S.M., Naimian, S., "Radioguided surgery using gamma detection probe technology for resection of cerebral glioma," Hellenic Journal of Nuclear Medicine, 18(Suppl 3). 68-75. 2015.

[11] Ormerod, I.E., McDonald, W.I., Du Boulay, G.H., Kendall, B.E., Moseley, I.F., Halliday, A.M., Kakigi, R., Kriss, A., Peringer, E., "Disseminated lesions at presentation in patients with optic neuritis," Journal of Neurology, Neurosurgery \& Psychiatry, 49(2). 124-7. 1986.

[12] Seddighi, A., Seddighi, A.S., Nikouei, A., Mohseni, G., "NS-06 Image Guided Surgery Using Neuronavigation System in Resection of Cerebral Gliomas Involving Eloquent Cortical Areas in Pediatric Population," Neuro Oncol, 18. 2016.

[13] Nakajima, H., Hosokawa, T., Sugino, M., Kimura, F., Sugasawa, J., Hanafusa, T., Takahashi, T., "Visual field defects of optic neuritis in neuromyelitis optica compared with multiple sclerosis," BMC neurology, 10(1). 45. 2010.

[14] Fazzone, H.E., Lefton, D.R., Kupersmith, M.J., "Optic neuritis: correlation of pain and magnetic resonance imaging," Ophthalmology, 110(8). 1646-9. 2003. 